\title{
Simulating High-Redshift Disk Galaxies: Applications to Long Duration Gamma-Ray Burst Hosts
}

\author{
Brant E. Robertson ${ }^{1,2,3}$ \\ ${ }^{1}$ Kavli Institute for Cosmological Physics, and \\ Department of Astronomy and Astrophysics, University of Chicago, \\ 933 East 56th Street, Chicago, IL 60637, USA \\ ${ }^{2}$ Enrico Fermi Institute, 5640 South Ellis Avenue, Chicago, IL 60637, USA \\ ${ }^{3}$ Spitzer Fellow \\ email: brant@kicp.uchicago.edu
}

\begin{abstract}
The efficiency of star formation governs many observable properties of the cosmological galaxy population, yet many current models of galaxy formation largely ignore the important physics of star formation and the interstellar medium (ISM). Using hydrodynamical simulations of disk galaxies that include a treatment of the molecular ISM and star formation in molecular clouds (Robertson \& Kravtsov 2008), we study the influence of star formation efficiency and molecular hydrogen abundance on the properties of high-redshift galaxy populations. In this work, we focus on a model of low-mass, star forming galaxies at $1 \lesssim z \lesssim 2$ that may host long duration gamma-ray bursts (GRBs). Observations of GRB hosts have revealed a population of faint systems with star formation properties that often differ from Lyman-break galaxies (LBGs) and more luminous high-redshift field galaxies. Observed GRB sightlines are deficient in molecular hydrogen, but it is unclear to what degree this deficiency owes to intrinsic properties of the galaxy or the impact the GRB has on its environment. We find that hydrodynamical simulations of low-stellar mass systems at high-redshifts can reproduce the observed star formation rates and efficiencies of GRB host galaxies at redshifts $1 \lesssim z \lesssim 2$. We show that the compact structure of low-mass high-redshift GRB hosts may lead to a molecular ISM fraction of a few tenths, well above that observed in individual GRB sightlines. However, the star formation rates of observed GRB host galaxies imply molecular gas masses of $10^{8}-10^{9} M_{\odot}$ similar to those produced in the simulations, and may therefore imply fairly large average $\mathrm{H}_{2}$ fractions in their ISM.
\end{abstract}

Keywords. Galaxies:high-redshift, galaxies:ISM, gamma rays: bursts

\section{Introduction}

To improve the physical description of star formation in hydrodynamical simulations of galaxies, Robertson \& Kravtsov (2008) implemented a new model for the ISM that includes low-temperature $\left(T<10^{4} \mathrm{~K}\right)$ cooling, directly ties the star formation rate to the molecular gas density, and accounts for the destruction of molecular hydrogen by an interstellar radiation field (ISRF) from young stars. They used simulations to study the relation between star formation and the ISM in galaxies and demonstrated that, for the first time, their new model simultaneously reproduces the molecular gas and total gas Kennicutt-Schmidt (KS) relations, the connection between star formation and disk rotation, and the relation between interstellar pressure and the fraction of gas in molecular form (e.g. Wong \& Blitz 2002, Blitz \& Rosolowsky 2006). The capability of this model to reproduce both the star formation efficiency and molecular abundance of nearby systems makes it useful for simulating low-mass galaxies that have suppressed $\mathrm{H}_{2}$ abundances (and whose star formation rates would be overestimated in common treatments of star 
formation based on the KS relation) and high-redshift galaxies whose structural properties may vary substantially from local systems (and may therefore not have the same KS relation normalization). The model should be especially useful for studying low-mass galaxies at high-redshift, such as long duration gamma-ray burst (GRB) host galaxies at $1 \lesssim z \lesssim 2$, which is the focus of this work.

The highly-energetic phenomena known as GRBs were discovered over forty years ago (Klebesadel et al. 1973), but their extragalactic origin was confirmed only in the last decade (e.g., Metzger et al. 1997). Since then, the properties of the cosmological population of galaxies that host GRBs have been increasingly well-studied (e.g., Bloom et al. 2002, Le Floc'h et al. 2006, Prochaska et al. 2006, Berger et al. 2007a,b). Recently, interest in long duration GRB galaxy hosts as possible tracers of the global star formation history of the universe has motivated systematic studies of their star formation efficiencies and stellar masses (Castro Cerón et al. 2008, Savaglio et al. 2008). These studies have found that high-redshift GRB hosts have small stellar masses $\left(\log M_{\star} \sim 9.3\right)$ and moderate star formation rates $\left(\mathrm{SFR} \sim 2.5 M_{\odot} \mathrm{yr}^{-1}\right)$. Compared with other high-redshift galaxy populations, GRB hosts tend to have lower star formation rates at fixed stellar mass compared with Lyman-break galaxies and lower stellar masses at fixed star formation rate compared with field galaxies (for details, see Savaglio et al. 2008).

Spectroscopic studies of GRB sightlines have provided additional information about the post-explosion character of the host galaxy ISM. Tumlinson et al. (2007) failed to detect $\mathrm{H}_{2}$ in five GRB sightlines and suggested that low metallicity and large far ultraviolet ISRF strengths $(10-100 \times$ the Milky Way value) were responsible for destroying molecular hydrogen in GRB hosts. They interpreted the lack of vibrationally excited $\mathrm{H}_{2}$ lines as evidence against the GRB destroying its parent molecular cloud, but noted various caveats to this conclusion such as the parent cloud size or cloud photodissociation before to the GRB. Whalen et al. (2008) used one-dimensional radiative hydrodynamical calculations to show that GRBs can ionize nearby neutral hydrogen, but suggested that an additional ISRF is necessary to remove molecular hydrogen from the nearby ISM. Prochaska et al. (2008) studied NV absorption in GRB sightlines, and argued that if nitrogen ionization by GRB afterglows leads to NV absorption then the observations support a scenario where dense, molecular cloud-like environments serve as the sites of GRBs.

Given the increasingly detailed studies of GRB hosts, their interesting ISM and star formation properties, and their low stellar masses, a theoretical study of GRB host galaxy analogues using hydrodynamical simulations that include a treatment of the molecular ISM is warranted. Below, we present simulations of a model GRB host galaxy that include a prescription for the molecular ISM and star formation in molecular clouds (Robertson \& Kravtsov 2008). We use the simulations to examine the star formation efficiency and molecular hydrogen content of galaxies with structural properties similar to those expected for low-mass galaxies at $1 \lesssim z \lesssim 2$. Below, we discuss our methodology and present some initial results.

\section{Methodology}

To study the properties of long duration GRB host galaxies, we simulate a numerical model of an isolated galaxy using a version of the N-body/Smoothed Particle Hydrodynamics code GADGET (Springel et al. 2001, Springel 2005b) that incorporates a model for the molecular ISM (Robertson \& Kravtsov 2008). For details regarding the numerical galaxy models, simulation methodology, and ISM model, we refer the reader to Springel 


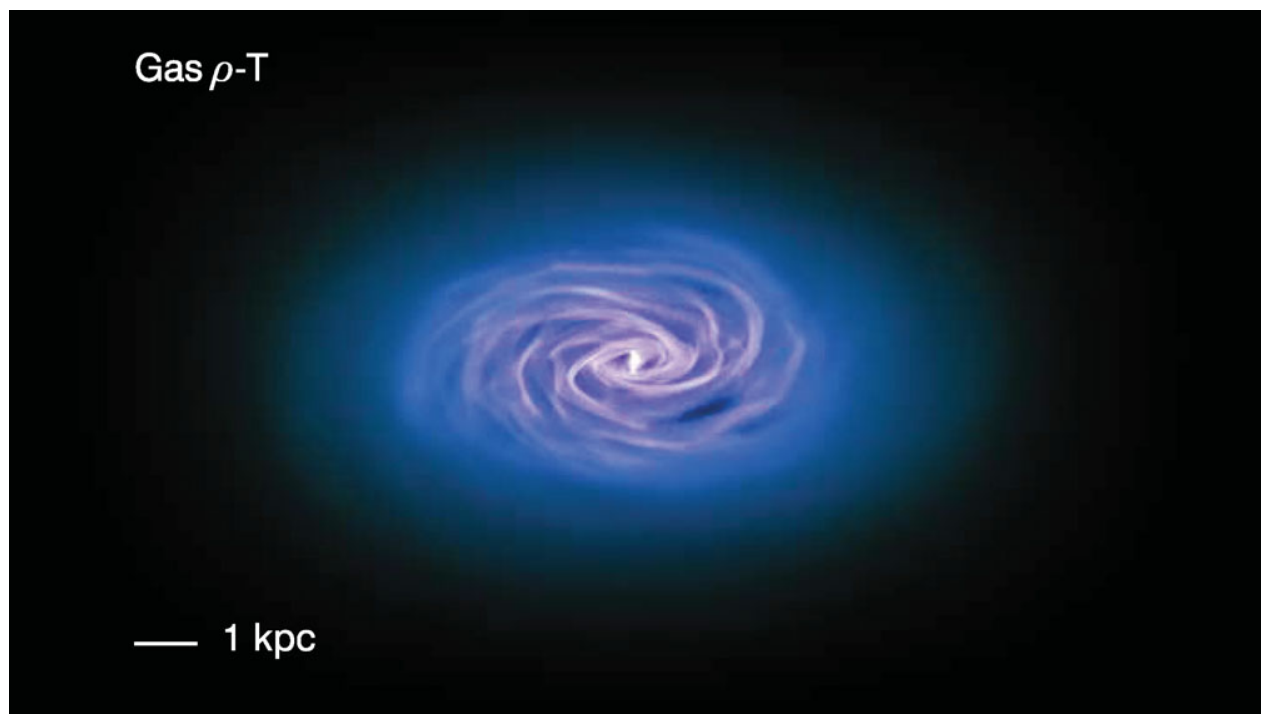

Figure 1. Simulated long duration Gamma-Ray Burst (GRB) host galaxy analogue at $z \sim 1.5$. Shown is the gas surface density of the GRB host (image intensity), color coded by the median interstellar medium temperature (purple regions have $T<10^{3} \mathrm{~K}$, while blue regions have $\left.T \gtrsim 10^{4} \mathrm{~K}\right)$. The simulated galaxy has a stellar mass $\log M_{\star} \approx 9.3$ and a star formation rate $\mathrm{SFR} \approx 1.2 M_{\odot} \mathrm{yr}^{-1}$, similar to high-redshift GRB host galaxies (e.g., Castro Cerón et al. 2008, Savaglio et al. 2008). The simulations include the Robertson \& Kravtsov (2008) model of the molecular ISM, enabling a study of the connection between star formation rate, galaxy properties, and $\mathrm{H}_{2}$ abundance in GRB hosts.

et al. (2005a), Robertson et al. (2006a,b), and Robertson \& Kravtsov (2008), but a brief summary follows.

The numerical galaxy model is designed to approximate the properties of $1 \lesssim z \lesssim 2$ GRB host galaxies as determined by Savaglio et al. 2008. The stellar disk mass of the system is set to $\log M_{\star}=9.3$, with a gas fraction of $f_{\text {gas }}=0.5$ (appropriate for highredshift, see Erb et al. 2006), which implies a total virial mass of $\log M_{\text {vir }}=10.9$ for a typical disk baryon fraction of $f_{\mathrm{b}}=0.05$. The virial radius is set appropriately for a halo with virial mass $M_{\text {vir }}$ at $z \sim 2$. The exponential disk scale length was fixed according to the Mo et al. (1998) formalism, including the adjustment for an effective Navarro et al. (1996) dark matter halo concentration of $c_{\mathrm{NFW}}=6$ (also appropriate for the chosen virial mass and redshift, see Bullock et al. 2001) and a spin of $\lambda=0.05$. The density field of the dark matter halo follows the Hernquist (1990) profile, while the velocity fields of the dark matter halo and the exponential stellar disk are set using the Hernquist (1990) distribution function and the epicyclical approximation, respectively. The numerical realizations of the stellar disk, gaseous disk, and dark matter halo are initialized with $N_{\text {disk }, \star}=4 \times 10^{5}, N_{\text {disk,gas }}=4 \times 10^{5}$, and $N_{\mathrm{DM}}=4 \times 10^{5}$ particles, and are evolved with a gravitational softening of $\epsilon=70 \mathrm{pc}$. The simulation is calculated for a duration of $t \sim 1$ Gyr, or about the time between redshift $z \sim 2$ and $z \sim 1.5$.

The simulation includes a treatment of the physics of the ISM and star formation following the model presented by Robertson \& Kravtsov (2008), and interested readers should examine that work for details. The photoionization code CLOUDY (Ferland et al. 1998 ) is used to tabulate the cooling rate, heating rate, molecular abundance, and related properties of gas as a function of density, temperature, metallicity, and local interstellar radiation field (ISRF) strength. The star formation rate is calculated by converting the 


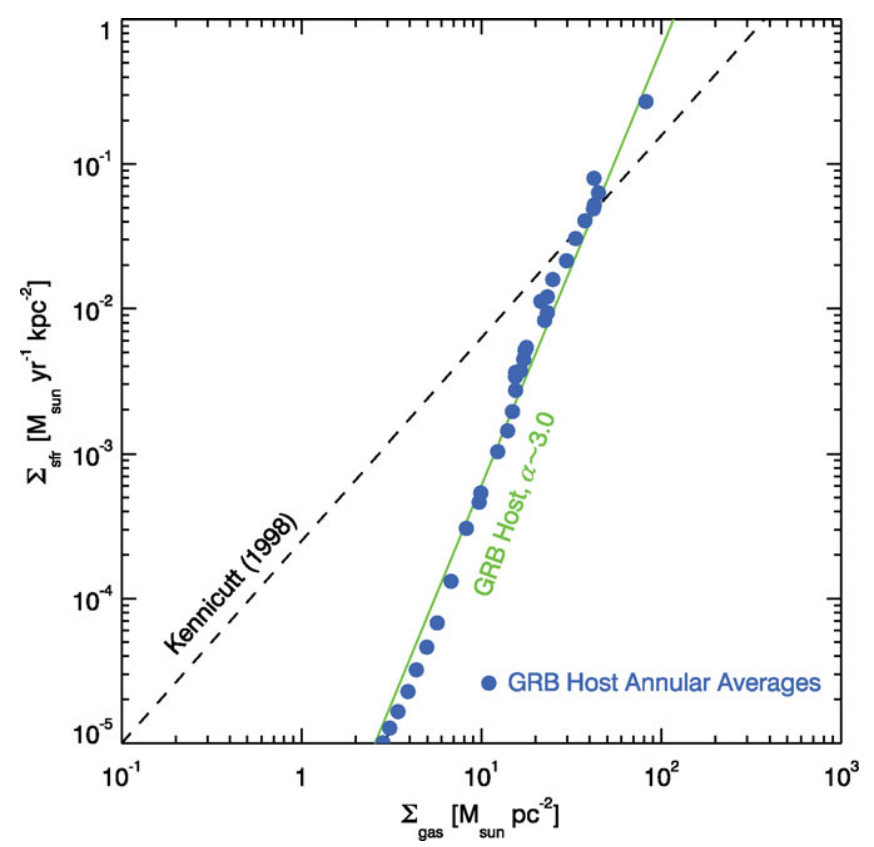

Figure 2. Kennicutt-Schmidt relation for a simulated GRB host galaxy at $z \sim 1.5$. Shown is the star formation rate surface density $\Sigma_{\mathrm{SFR}}$ as a function of total gas surface density $\Sigma_{\text {gas }}$, measured in annuli (blue dots). The average Kennicutt-Schmidt relation of the GRB host has a steeper power-law index $(\alpha \sim 3.0$, green line) than the disk-averaged relation measured by Kennicutt (1998; $\alpha \sim 1.4$, dashed line), owing to the suppression of $\mathrm{H}_{2}$ in the galaxy exterior by the interstellar radiation field and the low ISM metallicity (for a detailed discussion, see Robertson \& Kravtsov 2008).

molecular gas density to stars on a timescale that scales with the local dynamical time, with an efficiency set to match the star formation efficiency per free fall time in local molecular clouds (e.g., Krumholz \& McKee 2005, Krumholz \& Tan 2007). The local ISRF spectral shape is fixed to the local Milky Way ISRF inferred by Mathis et al. (1983), but the ISRF strength scales with the local star formation rate density (i.e., young, massive stars supply the local ultraviolet radiation field). The abundance of molecular gas tracked using CLOUDY includes the photodissociative and heating effects of this ISRF, and thereby includes a coarse accounting of the regulatory impact of the ISRF on star formation in molecular clouds.

\section{Results}

Figure 1 shows the gaseous structure of the GRB host galaxy model at $z \sim 1.5$ (after $900 \mathrm{Myr}$ of evolution). The figure shows the gas surface density of the system (image intensity) and the median temperature of the local ISM (purple regions have temperatures $T \lesssim 10^{3} \mathrm{~K}$, while blue regions have $T \gtrsim 10^{4} \mathrm{~K}$ ). The system has a rotational velocity of $v_{\text {rot }} \approx 100 \mathrm{~km} \mathrm{~s}^{-1}$ and a disk scale length of $R_{\mathrm{d}} \approx 1.5 \mathrm{kpc}$. During the simulation the star formation rate of the system varies in the range $\mathrm{SFR} \approx 0.5-2.5 M_{\odot} \mathrm{yr}^{-1}$, while the specific star formation rate is SFR $/ M_{\star} \approx 0.17-1.1 \mathrm{Gyr}^{-1}$. These properties are consistent with the properties of high-redshift GRB host galaxies determined by Savaglio et al. (2008), who find star formation rates of SFR $\sim 0.1-10$ and specific star formation rates of $M_{\star} / \mathrm{SFR} \sim 0.1-10 \mathrm{Gyr}$. 
The compactness of the system leads to a dense ISM and a considerable molecular gas fraction. The global, mass-weighted molecular abundance declines from $f_{\mathrm{H} 2} \sim 0.5$ at $z \sim 2$ to $f_{\mathrm{H} 2} \sim 0.3$ at $z \sim 1.5$. As a function of radius, the molecular fraction declines from $f_{\mathrm{H} 2} \sim 1$ near the center of the galaxy to $f_{\mathrm{H} 2} \sim 0.1-0.3$ beyond a disk scale radius. The typical star formation rate-weighted radius of the system is $r_{\mathrm{SFR}} \sim 0.8 \mathrm{kpc}$, where the ISM molecular fraction is $f_{\mathrm{H} 2} \sim 0.6$. Hence, if observed high-redshift GRB host galaxies are similar in nature to this simulated system, GRBs will likely occur in molecular-rich regions. While these molecular abundances are consistent with the spectroscopic studies by Prochaska et al. (2008), they are well above the observed $\mathrm{H}_{2}$ abundance along GRB sightlines (e.g., Tumlinson et al. 2007). In this model, the compact and dense structure of the high-redshift GRB host prevents the diffuse ISRF from suppress the $\mathrm{H}_{2}$ to levels observed in GRB sightlines. If an ISRF is responsible for suppressing $\mathrm{H}_{2}$ to observed levels in GRB sightlines, it may be generated by discrete point sources nearby the GRB in a manner not captured by the diffuse ISRF included in these simulations.

We note that in order to supply the star formation efficiency for GRB hosts determined by Savaglio et al. (2008), GRB hosts may need to be fairly molecule rich if their structure is similar to the simulated high-redshift galaxy analogues presented here. Figure 2 shows the total gas Kennicutt-Schmidt (KS) relation for the GRB host galaxy analogue, measured in annuli with a width of $\Delta r=100 \mathrm{pc}$. Plotted is the star formation rate density $\Sigma_{\mathrm{SFR}}$ as a function of the total gas surface density $\Sigma_{\text {gas }}$ (blue points), compared with the mean disk-averaged trend determined by Kennicutt (1998, dashed-line). The central concentration of molecular gas causes the total gas KS relation of the simulated GRB host galaxy analogue to be steeper than the disk-averaged relation. In order to supply the observed star formation rate of SFR $\sim 1-10 M_{\odot} \mathrm{yr}^{-1}$, as this simulated galaxy does, the typical consumption timescales of $\sim 100 \mathrm{Myr}$ for molecular gas imply a reservoir of roughly $M_{\mathrm{H} 2} \sim 0.1-1 \times 10^{9} M_{\odot}$ (the simulated system has $M_{\mathrm{H} 2} \sim 2-7 \times 10^{8} M_{\odot}$ during its evolution). Since observed GRB hosts have stellar masses of only $\log M_{\star} \sim 9.3$ (Savaglio et al. 2008), the inferred molecular fraction of the ISM should be large even for very gas rich systems.

Overall, we find that under standard assumptions about the mass and redshift scalings of galaxy structure hydrodynamical simulations of disk galaxies with stellar masses of $\log M_{\star} \sim 9$ that utilize a model for the molecular ISM and star formation in molecular clouds (Robertson \& Kravtsov 2008) can reproduce the observed star formation rates and efficiencies of GRB hosts (e.g., Castro Cerón et al. 2008, Savaglio et al. 2008). The star formation in both observed GRB hosts and the simulated GRB host analogue presented here is efficient for their low stellar masses, and to supply the observed range of star formation rates $\left(\mathrm{SFR} \sim 0.1-10 M_{\odot} \mathrm{yr}^{-1}\right)$ the molecular gas content such systems may need to be considerable $\left(f_{\mathrm{H} 2} \gtrsim 0.1\right)$. While this result is consistent with observations that suggest GRBs occur in dense, potentially molecular-rich regions of the ISM (e.g. Prochaska et al. 2008), more work is needed to reconcile such results with the low molecular abundance observed in GRB sightlines (e.g. Tumlinson et al. 2007) if GRBs cannot efficiently destroy $\mathrm{H}_{2}$ in the ISM (Whalen et al. 2008).

\section{References}

Berger, E., et al. 2007a, ApJ, 660, 504

Berger, E., et al. 2007b, ApJ, 665, 102

Bloom, J. S., et al. 2002, AJ, 123, 1111

Bullock, J. S., et al. 2001, MNRAS, 321, 559

Castro Cerón, J. M., et al. 2008, ApJ, submitted, arXiv:0803.2235 
Erb, D., et al. 2006, ApJ, 646,107

Ferland, G., et al. 1998, PASP, 110, 761

Hernquist, L. 1990, ApJ, 356, 359

Kennicutt, R. C. 1998, ApJ, 498, 541

Klebesadel, R. W., et al. 1973, ApJ (Letters), 182, L85

Krumholz, M. R. \& C. F. McKee. 2005, ApJ, 630, 250

Krumholz, M. R. \& J. C. Tan. 2007, ApJ, 654, 304

Le Floc'h, E., et al. 2006, ApJ, 642, 636

Mathis, J. S., et al. 1983, A\&A, 128, 212

Metzger, M. R., et al. 1997, Nature, 387, 878

Mo, H. J., et al. 1998, ApJ, 295, 319

Navarro, J., et al. 1996a, ApJ, 462, 563

Prochaska, J. X., et al. 2006, ApJ, 642, 989

Prochaska, J. X., et al. 2008, ApJ, accepted, arXiv:0806.0399

Robertson, B., et al. 2006a, ApJ, 641, 21

Robertson, B., et al. 2006b, ApJ, 641, 90

Robertson, B. \& A. V. Kravtsov . 2008, ApJ, 680, 1083

Savaglio, S., et al. 2008, ApJ, submitted, arXiv:0803.2718

Springel, V., et al. 2001, New Astron., 6, 79

Springel, V., et al. 2005a, MNRAS, 361, 776

Springel, V. 2005b, MNRAS, 364, 1105

Tumlinson, J., et al. 2007, ApJ, 668, 667

Whalen, D., et al. 2008, ApJ, accepted, arXiv:0802.0737

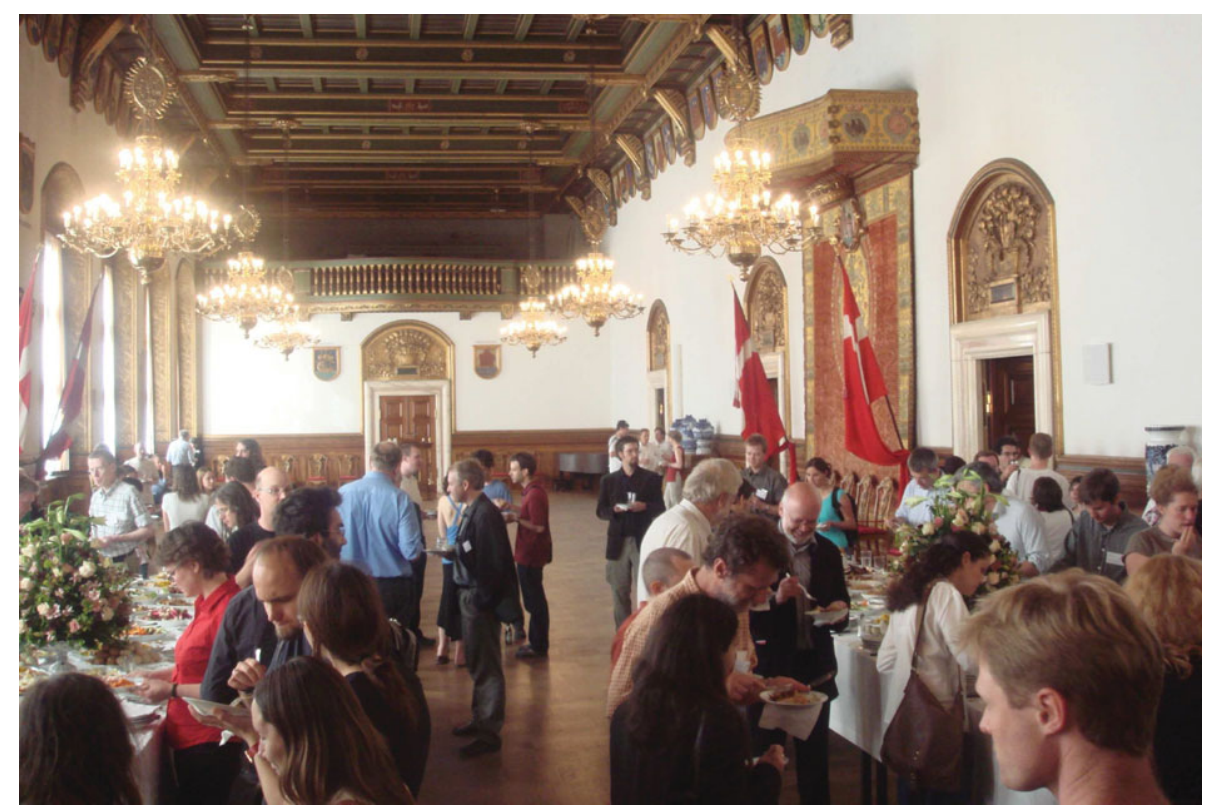

Particpants enjoying the reception at Copenhagen Town Hall. 